Presented at the Sixth International Conference on Ion Sources, Whistler, B.C., Canada, September 10-16, 1995, and to be published in the Proceedings

\section{RECEIVED}

JAN 241996

OSTI

Upgrade of a Vacuum Arc Ion Source Using a Strong Pulsed Magnetic Field

E.M. Oks, I.G. Brown, M.R. Dickinson, and R.A. MacGill

August 1995

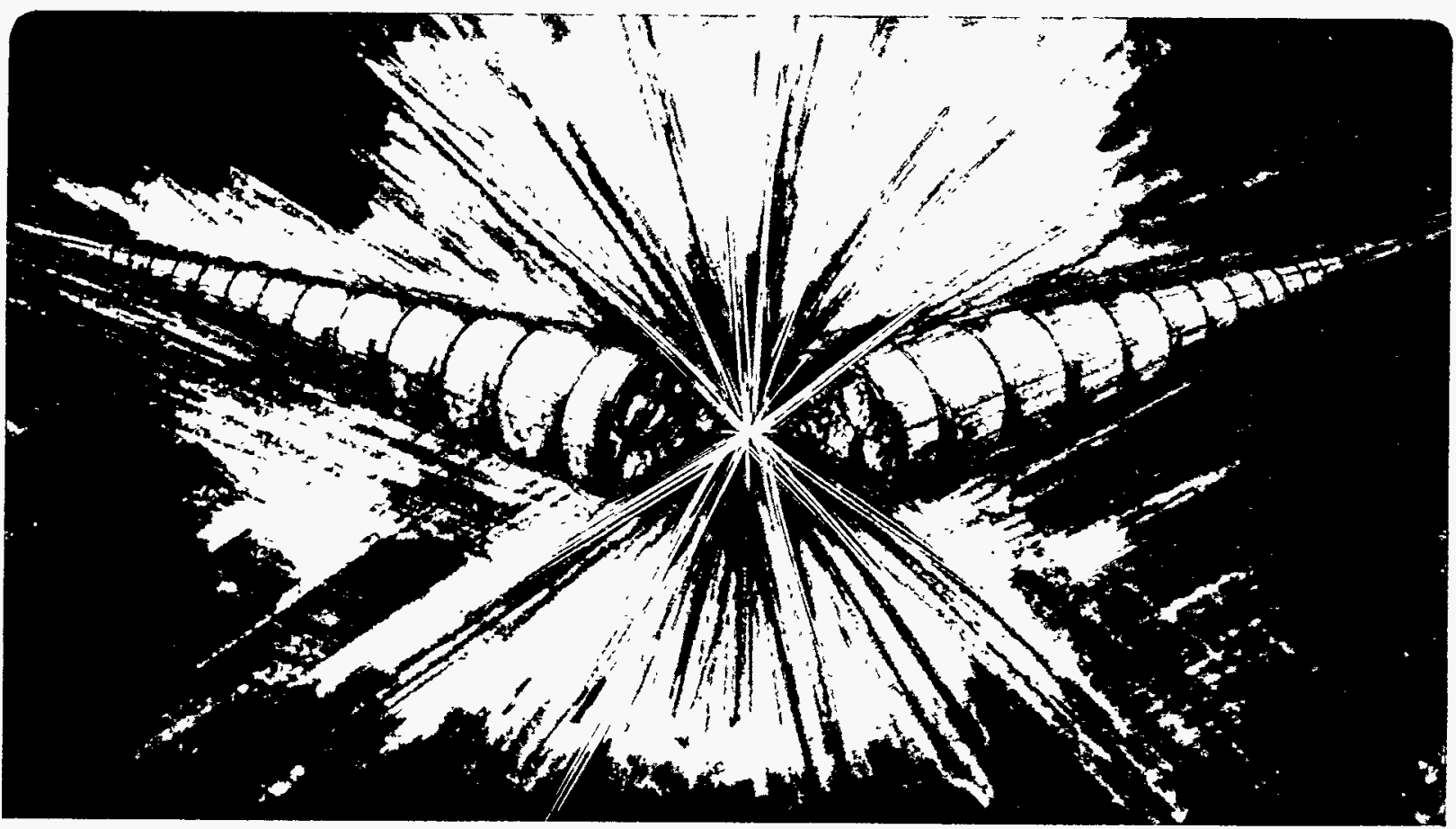

Prepared for the U.S. Department of Energy under Contract Number DE-AC03-76SF00098 


\section{DISCLAIMER}

This document was prepared as an account of work sponsored by the United States Government. While this document is believed to contain correct information, neither the United States Government nor any agency thereof, nor The Regents of the University of Califomia, nor any of their employees, makes any warranty, express or implied, or assumes any legal responsibility for the accuracy, completeness, or usefulness of any information, apparatus, product, or process disclosed, or represents that its use would not infringe privately owned rights. Refcrence herein to any specific commercial product, process, or service by its trade name, trademark, manufacturer, or otherwise, does not necessarily constitute or imply its endorsement, recommendation, or favoring by the United States Government or any agency thereof, or The Regents of the University of California. The views and opinions of authors expressed herein do not necessarily state or reflect those of the United States Government or any agency thereof, or The Regents of the University of Califomia.

Lawrence Bcrkeley National Laboratory

is an equal opportunity employer. 
LBL-37221

UC-426

\title{
Upgrade of a Vacuum Arc Ion Source Using a Strong Pulsed Magnetic Field
}

E.M. Oks, I.G. Brown, M.R. Dickinson, and R.A. MacGill

\author{
Accelerator and Fusion Research Division \\ Lawrence Berkeley Laboratory \\ University of California \\ Berkeley, California 94720
}

August 1995

This work was supported in part by the Director, Office of Energy Sciences, Advanced Energy Projects Division, of the U.S. Department of Energy under Contract No. DE-AC03-76SF00098, and by the Electric Power Research Institute under Award Number 8042-03. 
8 royved pape 


\title{
UPGRADE OF A VACUUM ARC ION SOURCE \\ USING A STRONG PULSED MAGNETUC EIELD
}

\author{
E.M. Oks \\ High Current Electronics Institute, Russian Academy of Sciences, and \\ State Academy of Control Systems and Radioelectronics, Tomsk 634050, Russia \\ I.G. Brown, M.R. Dickinson and R.A. MacGill \\ Lawrence Berkeley Laboratory, University of California, Berkeley, CA 94720, USA
}

\begin{abstract}
A pulsed magnetic field of up to $10 \mathrm{kG}$ was incorporated into a vacuum arc ion source. The field was established by a small coil surrounding the arc discharge region, powered by either an additional power supply (capacitor bank) or by the arc power supply (arc current and coil current in series). This addition has led so a number of improvements in source performance: the mean charge state of the metal ions produced was enhanced by a factor of up to two, for 30 different cathode materials from carbon to bismuth; hybrid metal / gaseous ion beams could be generated when an additional gas (nitrogen, oxygen or argon) was admitted into the source, with gaseous ion fraction as high as $50 \%$; triggering of the source could be done by a very long lifetime gaseous pre-discharge technique. We also report on the use of a wire mesh to stabilize the plasma emission surface at the extractor as a means for achieving a flat beam current characteristic as a function of extraction voltage.
\end{abstract}




\section{INTRODUCTION}

Magnetic fields are widely used in ion source application [1] as a means of confining the discharge electrons and providing better ionization. For field strengths of a few hundred gauss, the field is adequate to magnetize the discharge electrons only. Increasing the magnetic field up to the levels needed for ion confinement can be difficult because of the power needed to establish the field over the large discharge volume, and because of major perturbations to the ion beam formation process at the extractor.

For vacuum arc ion sources it has been shown that a magnetic field of a few hundred gauss can serve to increase the ion beam current $[2,3]$, improve the beam current density uniformity [4], reduce the beam noise [5], and permit hybrid metal/gaseous ion generation [6]. In contrast to other kinds of discharges, in vacuum arcs all of the processes and phenomena which determine the plasma parameters take place in a rather narrow region close to the cathode surface, at the cathode spots. So in principal the magnetic field need be of small volume and only in the arc region near the cathode. This allows for the possibility of employing a strong magnetic field in vacuum arc sources without the problems mentioned above. Here we present experimental results concerning the effect of a strong magnetic field on the vacuum arc ion source parameters.

\section{EXPERIMENTAL SET-UP}

All of the experiments were carried out using the LBL $10-\mathrm{cm}$ extractor vacuum arc ion source, modified by the addition of a small pulsed coil as described. Beam current was measured with a downstream Faraday cup, and ion charge state distribution was monitored by a time-of-flight system. For the experiments described here, the source was pulsed at a low duty cycle of about 1 pulse per second. Base pressure was typically in the low $10^{-6}$ Torr range. The complete set-up has been fully described elsewhere [7,8]. 


\section{RESULTS}

\section{A. Ion charge state enhancement}

For a magnetic field of a few kilogauss there was a significant increase in the high charge state fractions in the vacuum arc plasma and ion beam. We have investigated about 30 different cathode materials and measured the enhancement in mean charge state. The ratio of mean charge states with and without magnetic field varies from 1.2 for tungsten up to about 1.9 for most cathode materials and to 2.5 for bismuth. An example of this influence for an $\mathrm{Fe}$ cathode is shown in Figure 1. One can see that the singly-ionized fraction decreases, the medium charge state fractions $(2+$ and $3+$ ) have optimal values of magnetic field for which they separately maximize, while the highest charge state (4t) increases with increasing B field. There are at least three reasons for this: higher electron temperature, higher plasma density, and longer ion lifetime in the discharge. More detailed discussion on the elevation of ion charge states in a strong magnetic field has been presented elsewhere [8-10].

\section{B. Generation of gaseous ions}

Related work on the production of gaseous ion species in vacuum arc ion sources has been carried out collaboratively with GSI (Darmstadt, Germany), and this work has been reported in detail [6]. In this prior work, we investigated a large number of different cathode materials and a wide range of arc current $(100 \mathrm{~A}-4 \mathrm{kA})$ and magnetic field strength $(10 \mathrm{mT}$ $2 \mathrm{~T}$ ). As described in [6], the presence of any kind of gas with any cathode material always leads to a decrease of the more highly stripped metal ion fractions in the vacuum arc plasma and to an increase of the low charge state fractions. The magnetic field helps in gaseous ion generation too.

An example of the kind of results obtained is shown in Figure 2. One can see that the presence of gas shows an influence starting at very low pressure. First the $Q=4+$ fraction begins to decrease, then $3+$, while the $1+$ and $2+$ components rise. We also observed that for 
high gas pressure the total ion current that is extracted from the vacuum arc plasma is decreased. As can be seen in Figure 2, the gaseous ion fraction can be as great as $50 \%$ or more. For molecular gases like oxygen or nitrogen, both atomic and molecular ion species are observed, and their relative proportions are dependent on magnetic field; with increasing field strength the atomic ion fraction increases and the molecular component decreases; see Figure 3.

Simple estimates show that the particle density of the added gas is comparable to the particle density of metal vapor rather far from the cathode spot (more than $1 \mathrm{~mm}$ ), and therefore the feed gas has no direct influence on the primary processes of charge state formation which take place at the cathode spots. Never-the-less the influence of gas on the charge state distribution is significant.

Thus the presence of gas can change the charge state composition of the metal ion beam as well as allowing for mixed species beams. Interest in this operating mode for ion implantation application of course has to do with the ability to form buried layers of mixed composition such as, for example, oxides and nitrides.

\section{C . Arc ignition by a gaseous pre-discharge}

In the presence of a strong magnetic field the vacuum arc trigger system based on discharge across an insulating surface can be replaced by a gaseous discharge trigger. This kind of triggering system has been used previously in a number of arc devices [11]. For the vacuum arc metal ion source the problem is to obtain stable ignition with the background gas pressure as low as possible, because of the strong influence of the added gas to the ion beam parameters. In order to operate at low pressure we chose to use crossed electric and magnetic fields with oscillating electrons. An additional pressure decrease was achieved by operating with a very high magnetic field of up to $10-15 \mathrm{kG}$. 
We used both Penning [12] and magnetron gas discharges. The experimental set-up based on an "inverse magnetron" system is shown in Figure 4. The discharge unit is formed by two cylindrical electrodes: trigger and cathode with diameters $3 \mathrm{~mm}$ and $18 \mathrm{~mm}$ respectively. The vacuum arc is ignited between cathode and hollow anode. There are three pulsed power supplies: magnetic field $(300 \mathrm{~V}, 500 \mathrm{~A}, 0.5 \mathrm{~ms})$, trigger discharge $(1-10 \mathrm{kV}$, $50 \mathrm{~A}, 10 \mu \mathrm{s})$, and main $\operatorname{arc}(500 \mathrm{~V}, 500 \mathrm{~A}, 0.25 \mathrm{~ms})$. Only the magnetic field supply uses an electronic switch; the other two are connected to the discharge electrodes directly. The magnetic field is switched on first and then the magnetron discharge is started. Because of the high current, cathode spots are formed and then the main arc discharge follows. As indicated in Figure 5, at a pressure of $4 \times 10^{-6}$ Torr a $9 \mathrm{kV}$ trigger was necessary for reliable magnetron ignition. Steady and stable vacuum arc triggering was obtained. It is important to note that for this low background gas pressure the influence of gas on the vacuum arc ion source parameters is negligible.

\section{Grid stabilization of the plasma emission surface}

For high magnetic field strength the plasma density and plasma ion current density are high also. When the plasma density is too high, beamlet formation at the accel-decel beam formation electrodes can be poor (failure of appropriate plasma meniscus to form, and penetration of the high density plasma through the beamlet apertures), leading to defocusing of the ion beam and significant loss of beam to the extraction electrodes. On the other hand, if the extraction voltage is too high for a given plasma density then over-focusing of the beamlets can occur and again poor quality overall beam can result. In principle a multiaperture accel-decel extraction system is optimum for just one set of plasma and extraction parameters (geometry, accelerating voltage, and current density). Often this is an inconvenience.

One way to solve this problem is by grid stabilization of the plasma surface, as has been used widely for plasma electron sources [13] and can be applied for ion sources [14]. The 
idea is to form an emission electrode on the plasma side of the first extractor grid by means of a grid with very small holes and high transparency. Because of the small hole diameter the high voltage accelerating field will not penetrate through the holes into the plasma region, and thus the plasma emission surface (meniscus) will not change its shape with increasing extraction voltage. The results of measurements made with and without grid are shown in Figure 6. One can see a saturation with the grids and decrease of current at high voltage because of over-focusing. This allows a more stable and reliable high voltage source operation.

This work was supported in part by the Electric Power Research Institute under Award No. 8042-03 and the U.S. Department of Energy, Office of Basic Energy Sciences, under Contract Number DE-AC03-76SF00098. One of us (EMO) was supported at LBL under the DOE ILAB U.S.-Russia collaborative research program. 


\section{References.}

1. The Physics and Technology of Ion Sources, edited by I.G. Brown (Wiley, New York,1989).

2. I.G. Brown, Nucl. Instrum. Meth. Phys. Res. B37/38, 68 (1989).

3. S.P. Bugaev, A.G. Nikolaev, E.M. Oks, P.M. Schanin and G.Yu. Yushkov, Rev.Sci.Instrum. 63, 2224 (1992).

4. S.P. Bugaev, A.G. Nikolaev, E.M. Oks, P.M. Schanin and G.Yu. Yushkov Rev.Sci.Instrum. 65, 3119 (1994).

5. E. Oks, P. Spädtke, H.Emig and B.H. Wolf, Rev.Sci.Instrum. 65, 3109 (1994).

6. P. Spädtke, H. Emig, B.H. Wolf and E. Oks, Rev.Sci.Instrum. 65, 3113 (1994).

7. I.G. Brown, Rev. Sci. Instrum. 10, 3061 (1994).

8. E.Oks, I.G. Brown, M.R. Dickinson, R. MacGill, H.Emig, P.Spädtke and B.H. Wolf, Appl. Phys. Lett. 67, 200 (1995).

9. F.J. Paoloni and I.G. Brown, Rev. Sci. Instrum. 66, 3855 (1995).

10. P. Spädtke, H. Emig, B.H. Wolf and E. Oks, GSI Scientific Report 1994 (GSI 95-1), Darmstadt, Germany, March 1995, p.227.

11. N.V. Gavrilov, N.N. Koval, Yu.E. Kreindel, E.M. Oks and P.M. Schanin, Nucl. Instrum. Meth. Phys. Res. A312, 417 (1992).

12. A.G. Nikolaev, P.M. Schanin, G.Yu. Yushkov and E.M. Oks, 22nd Int. Conf. on Phenomena in Ionized Gages, July 31 - August 4, 1995, Hoboken, New Jersey, USA, p. 1-95.

13. E.M. Oks, Plasma Sources Sci.Technol. 1, 249 (1992).

14. S. Humphries, Jr., C. Burkhart and L.K. Len, in The Physics and Technology of Ion Sources, edited by I.G. Brown (Wiley, New York, 1989), p. 397. 


\section{Figure captions}

Fig. 1 Charge state fractions as a function of arc current and magnetic field strength for an iron plasma and ion beam. $\mathrm{B} / \mathrm{I}=10 \mathrm{G} / \mathrm{A}$.

Fig. 2 Ion current fractions in a mixed Pt-N beam as a function of nitrogen gas pressure.

Fig.3 Oxygen ion fractions as a function of magnetic field and arc current. $B / I=10 \mathrm{G} / \mathrm{A}$, $\mathrm{P}=2 \times 10^{-4}$ Torr.

Fig.4 Schematic of vacuum arc ignition system based on "inverse magnetron discharge".

Fig.5 Magnetron discharge ignition voltage as a function of nitrogen gas pressure.

Fig.6 Current-voitage characteristic of ion source with and without wire mesh at the first extractor electrode. 


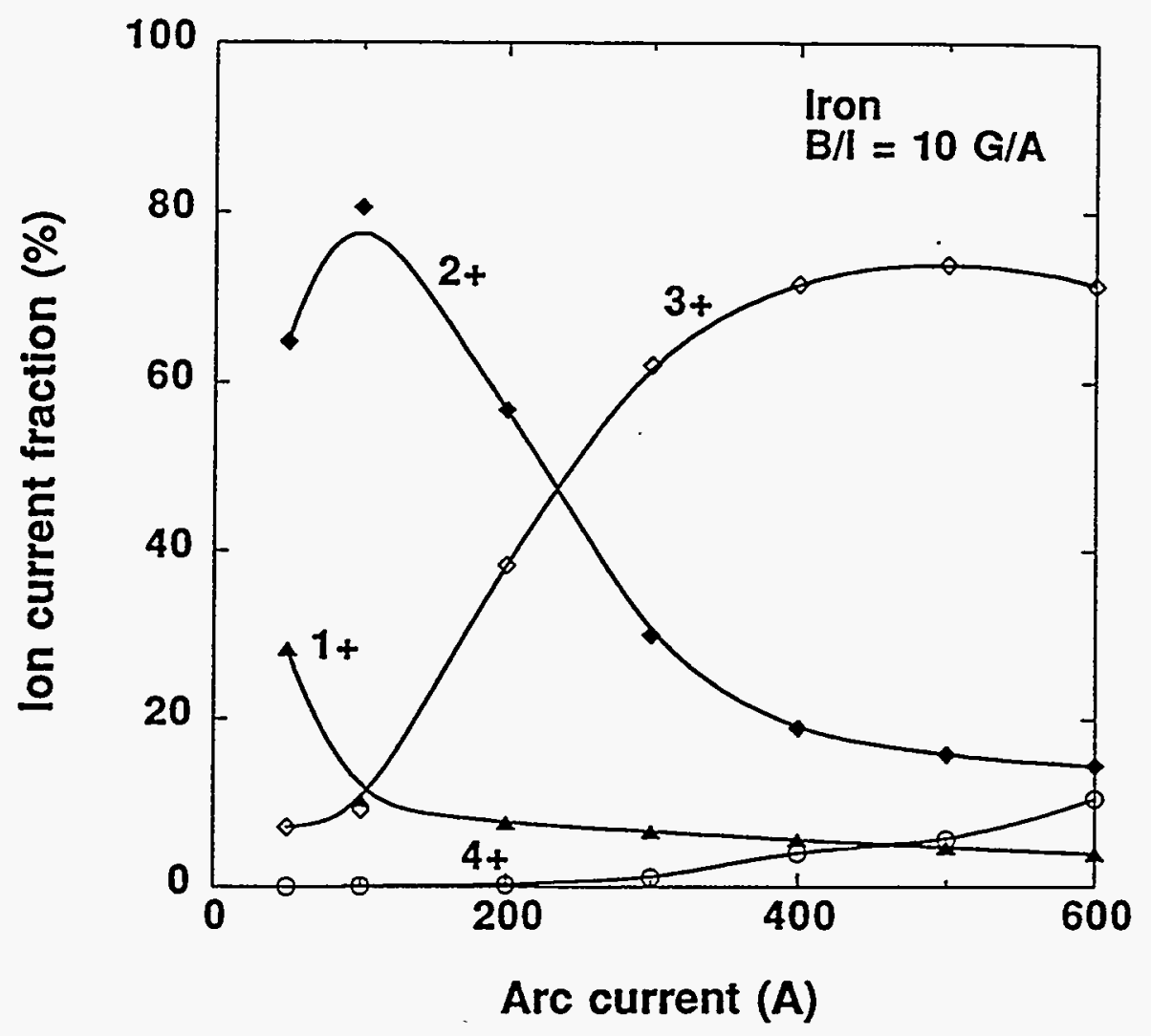

Fig. 1 


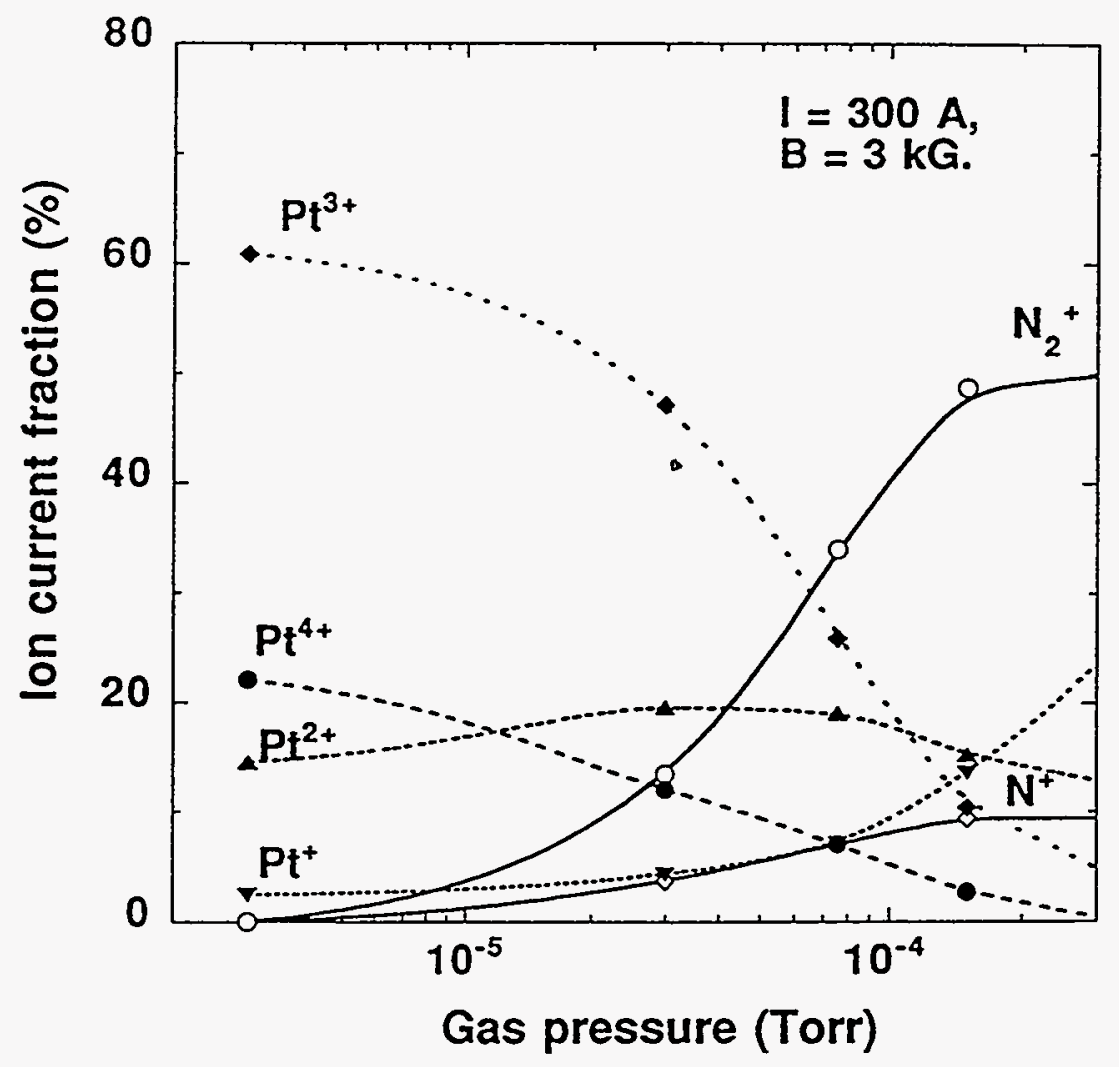

Fig. 2 


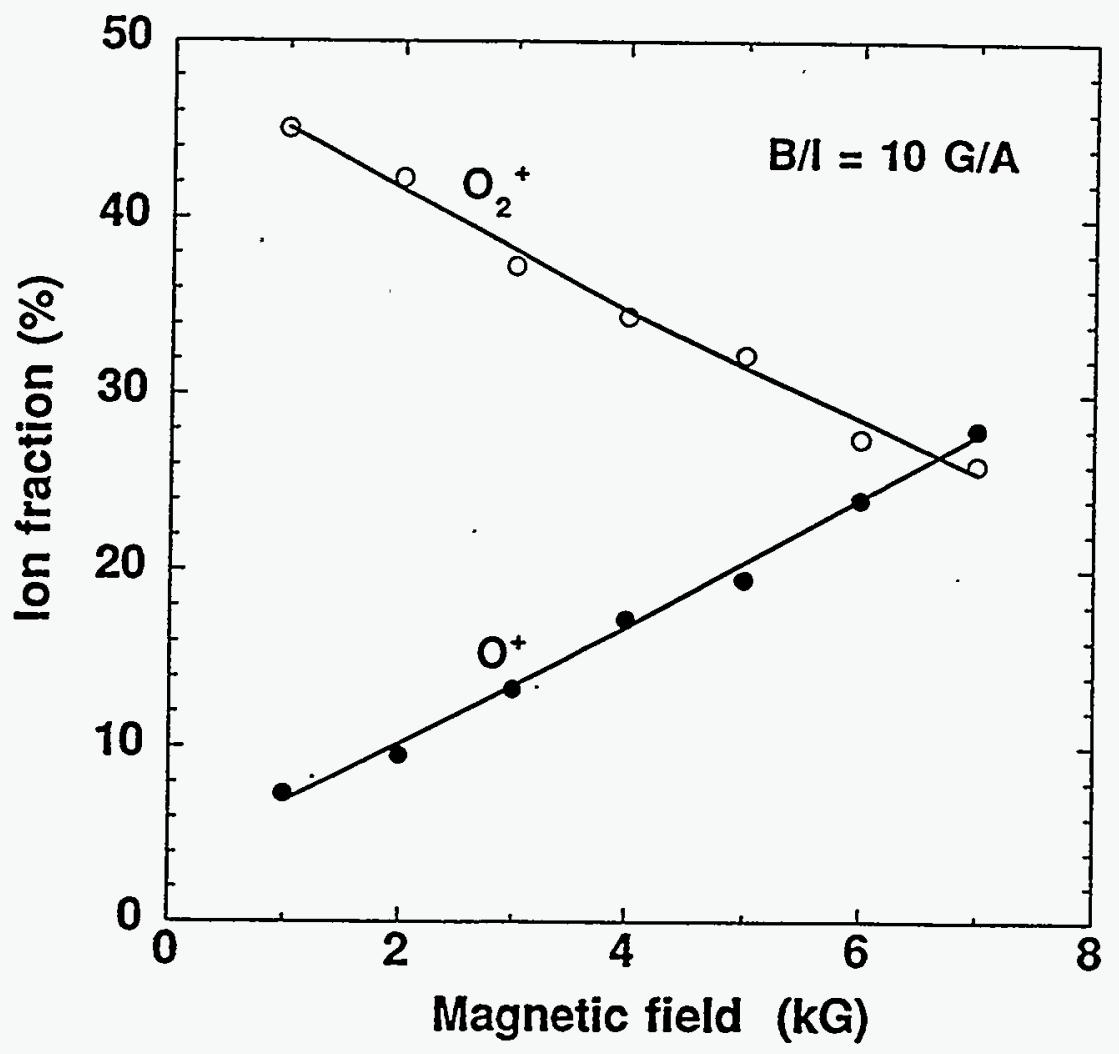

Fig. 3 


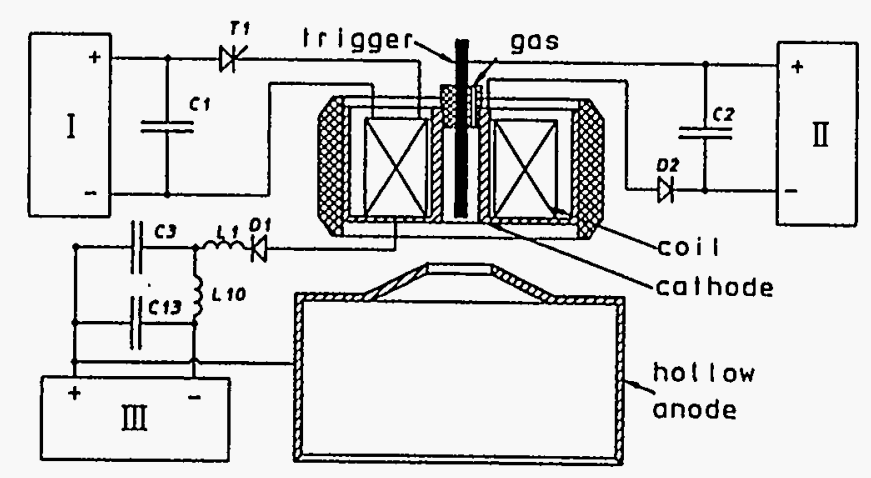

Fig. 4 


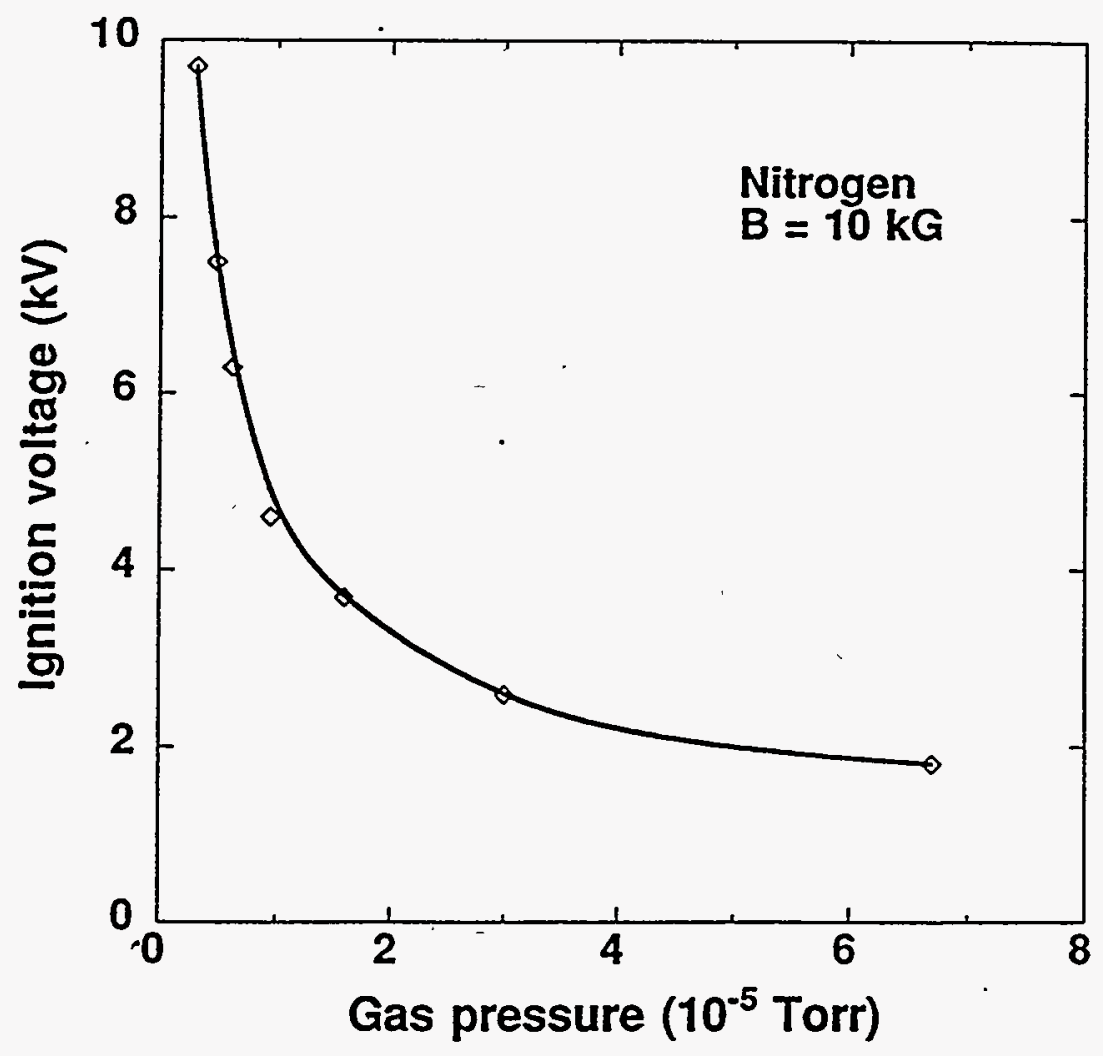

Fig. 5 


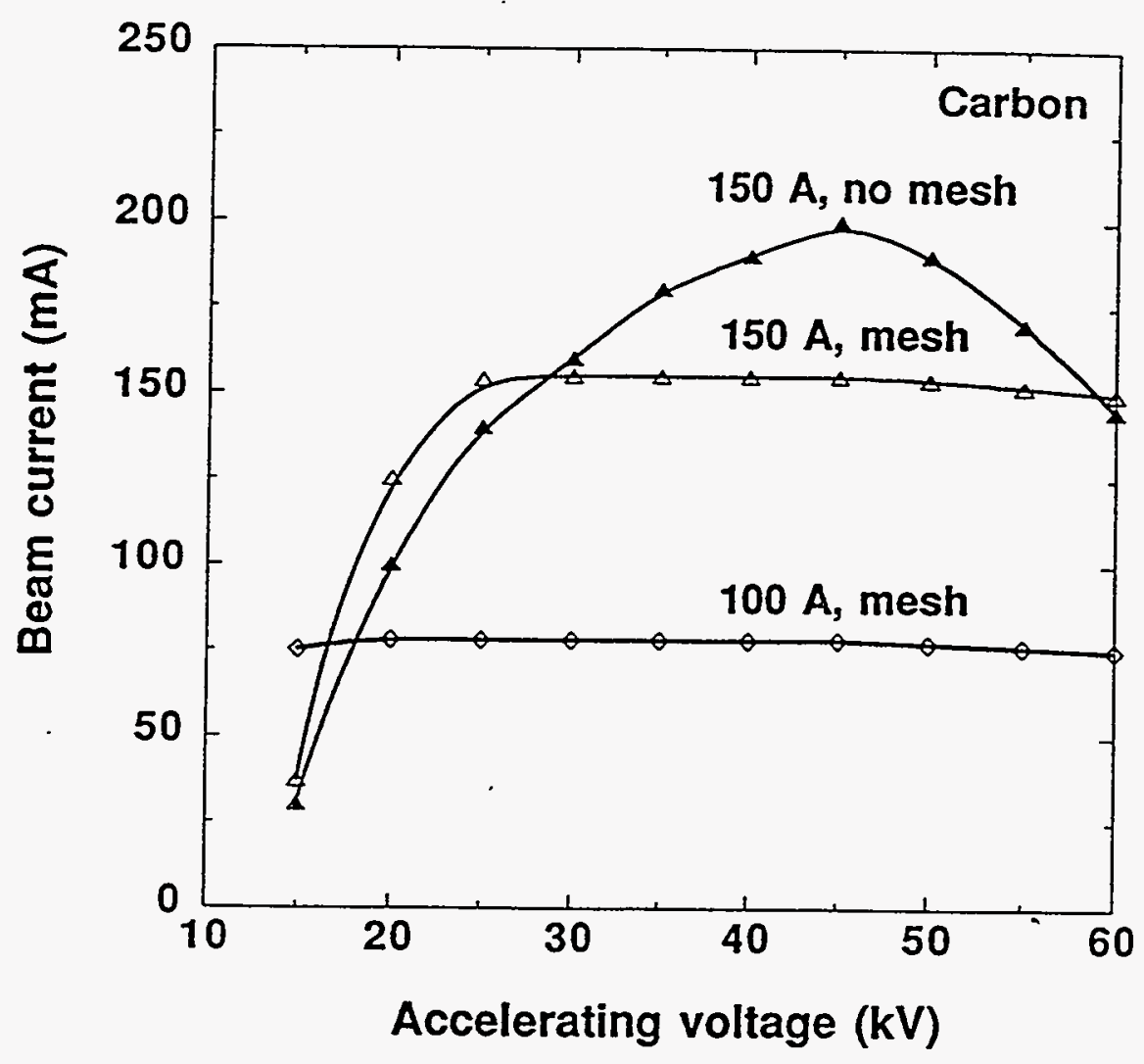

Fig. 6 